\title{
Developing an online learning community through an open reflective assessment
}

\author{
John Owen, Catherine Wasiuk, Ailsa Nokes, Sian Roderick \\ The University of Manchester, UK
}

\begin{abstract}
The concepts of learning communities, open educational practices and co-created teaching and learning are topics of current debate, particularly since the switch to online learning in 2020/21 because of the COVID-19 pandemic. This case study uses student feedback to evaluate a new assessment method introduced within an online distance learning Master of Public Health programme. The assessment required students to blog about their motivations for studying public health, submitting their reflections to an open online platform, resulting in the co-creation of a shared, open-learning resource for current and future students. The assessment design was informed by the benefits of open educational practices and co-created teaching and learning, with the overall aim of developing an online learning community that will continue to grow and develop beyond the initial assessment and cohort. Feedback suggests that the open, reflective nature of the assessment had a positive impact on the student learning experience and contributed towards a sense of learning community through enhanced social cohesion within the group.
\end{abstract}

Keywords: learning communities, open educational practices, co-created resources, reflective assessment

\section{Background}

The University of Manchester Master of Public Health (MPH) is a global, online, distance-learning programme. Traditionally, most students studying for the MPH already work in public health or in areas relating to it. However, over recent years, the programme has attracted interest from a broader range of students from non-traditional public health backgrounds. Responding to the needs of these students, a new course unit, 'Introduction to Public Health', was added to the programme in September 2020. This presented an exciting opportunity to design and implement an authentic learning experience for students new to the subject, including open educational practices and cocreated resources. The benefits of active learning communities were also considered during the design of the course unit's formative and summative assessments, resulting in new assessment methods not used before on the MPH programme. This case study evaluates one of these new assessment methods.

Introduced in the first week of the course unit, one such assessment method required students to write a reflective blog post about their motivations for studying public health. The reflective writing assessment was a 600-word blog post worth ten per cent of the unit marks and assessed using equally weighted criteria for clarity, relevance, analysis, interconnections and self-criticism. Once they had written their blog post, the students were encouraged to publish it on an open platform ('Medium'), with the option to submit it via the virtual learning environment ('Blackboard') if they felt uncomfortable about publishing it on Medium. After publishing their reflections, students could also 
submit them to an online open publication called 'Why Public Health?' that was created specifically for this assessment on Medium.

The aim of the assessment was to

- introduce reflective writing early in the course unit

- share authentic student experiences with peers on an open platform

- present an opportunity for co-creation of teaching and learning to benefit current and future cohorts

- develop the foundations of a learning community for the current cohort.

The reflective blog complemented a range of activities in the course unit aimed at encouraging student-student engagement and group cohesion. These included a 'Padlet' map board for students to introduce themselves (background, interests and location) and four formative discussion board questions relating to each of the four topics. A summative group project also presented an opportunity for students to work in small teams on a health communication project of their choosing.

The case study draws on current literature to highlight the benefits and challenges of implementing the personal, reflective assessment through an open online platform and to demonstrate how the approach can form the basis for learning communities through co-created, student-generated content.

\section{Implementation}

The assessment required students to reflect on 1) how a personal event or experience challenged their assumptions and biases in the context of health and wellbeing and 2) how this motivated them to study public health. It was anticipated that introducing reflective writing early in the course unit might help students get to know each other - by sharing their personal reflections on an open platform - and present students working within their curriculum with opportunities to co-create it. Bovill and Woolmer (2018) define co-creation in the curriculum as "co-design of learning and teaching within a course or programme usually during the course or programme", and co-creation of the curriculum as "co-design of a programme or course, usually before the programme or course takes place". Students studying Introduction to Public Health would not only be creating and sharing their own content and learning during the course; they would also be developing content and potential learning materials for future student cohorts. Furthermore, Johnston and Stewart (2020) argue that the concept of open pedagogy can help provide a meaningful foundation for student-staff partnerships. They suggest that open pedagogy and the 'students-as-partners' approach are both "high-impact practices focused on authenticity, community, and responsibility" (Healey et al., 2014, p.82). This supports the approach taken during the conceptualisation, planning and design of the new course unit and particularly its authentic, student-centred assessments.

Medium was selected as the platform for the assessment. Medium is an online publishing platform enabling individual authors to write a 'story' (now referred to as 'blog posts'). It has the additional functionality of publications - collections of related blog posts by different authors. Students were instructed to submit their reflection on Medium, but, if they felt uncomfortable about publishing on an open platform, they could either write under a pseudonym or upload a Word document privately to an assignment on Blackboard. If students chose to publish their blog post on Medium, they could 
also submit - as an optional step, not a compulsory assessment requirement - to the 'Why Public Health?' publication.

The assessment was designed to create a shared, communal, open-learning resource (Bonk, 2012; Blake et al., 2019), so departing from the common practice of submitting individual assessments on closed institutional platforms like Blackboard. This is an example of how the use of technology in education can create pedagogical innovation (Conole, 2013, p.52) by "providing new ways of collaborative creation and exchange of learning content" and establishing "new forms of communication amongst learners and teachers." The assessment design has the potential for enhanced social cohesion and the development of an online learning community through open, critical reflection in a public space (Conrad, 2005; Kear, 2010; Kear et al., 2014).

\section{Methods}

The new course unit, 'Introduction to Public Health', ran for the first time in September 2020. There were twenty-five students on the course unit. Most were based in the United Kingdom (UK), with two from Ireland and three from sub-Saharan Africa. Generally, the students who chose this course unit were from a diverse range of non-traditional public health backgrounds and were often returning to education after more than five years. Feedback on this new method of assessment was gathered from several sources.

\section{Survey}

An online survey was distributed to the cohort to gather feedback on writing about a personal experience on an open online platform. The survey consisted of a combination of ten open and closed questions with the opportunity for free-text comments to elaborate on given answers; one of these questions offered a list of statements and asked students to say how far they agreed or disagreed with them (see appendix 1). The aim of the survey was to analyse how students felt before the assessment and how their feelings changed after it, as well as to draw on the positives and negatives of this assessment type, with specific reference to the open nature of the assessment, authentic learning and social cohesion as an online group.

\section{Additional evaluation processes}

Feedback was also gathered from other channels throughout the course unit, including the standard end-of-course-unit survey, the online discussion boards in Blackboard and a topic feedback form. The discussion boards and the topic feedback form were always open to the students. They did not directly ask the students for feedback on the assessment, though some responses specifically mentioned the blog).

\section{Evaluation}

\section{Results}

All students $(n=25)$ on the course published their reflections on Medium. No student took the option to submit a Word document to Blackboard, but two students did publish their blog post under a pseudonym. Fourteen students chose to submit to the publication 'Why Public Health?'. 


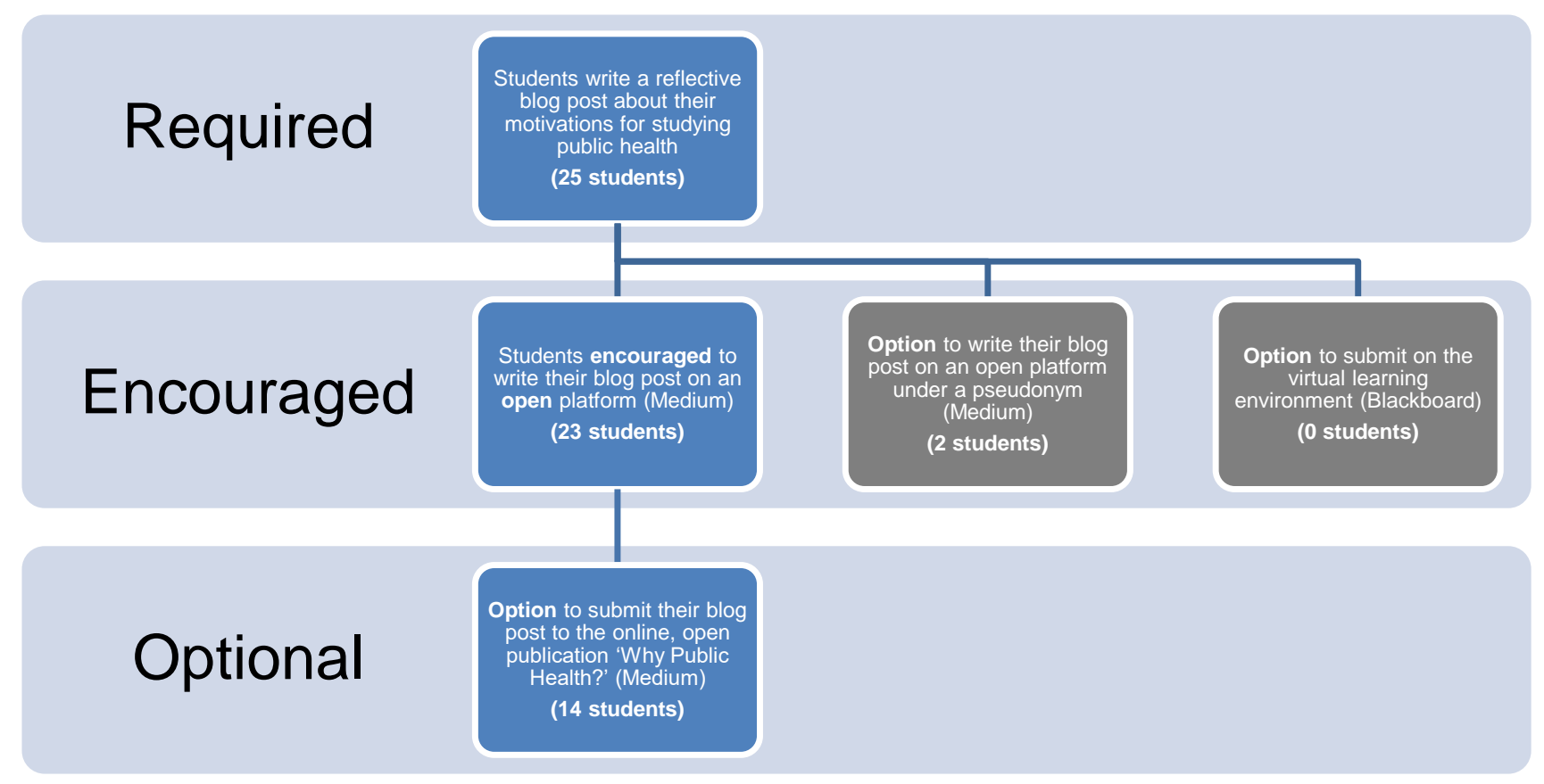

Figure 1: The four publishing options for the assessed blog post

Table 1: Feedback on the assessment was obtained in the following formats:

\begin{tabular}{|c|c|}
\hline Method & Number of respondents \\
\hline \multicolumn{2}{|l|}{$\begin{array}{l}\text { Direct feedback (questions specifically } \\
\text { asked about the blog assessment) }\end{array}$} \\
\hline Survey & 10 \\
\hline \multicolumn{2}{|c|}{$\begin{array}{l}\text { Indirect feedback (specifically mentioning } \\
\text { blog assessment / reflective writing) }\end{array}$} \\
\hline End-of-course-unit survey & 4 \\
\hline Online discussion boards in Blackboard & 3 \\
\hline End-of-topic feedback form & 4 \\
\hline
\end{tabular}

\section{Discussion}

In the survey, students were asked how they felt writing about a personal experience on an open online platform. Four students said they 'didn't feel any apprehensiveness writing about a personal experience on an open online platform'; four students 'had no problems writing about a personal experience, but were concerned about posting it on an open online platform'; two students were 'mostly worried writing about a personal experience rather than posting on an open online platform'. 
Of those responses that were mostly concerned about writing on an open platform, the main issues related to how others would react to their blog posts, either from a professional or personal perspective. One student was concerned about the potential for their employer or clients to read and perhaps misinterpret their words and another did not want their name to be attributed to their blog post. One student said that they had Googled their name and it had appeared high in the search results, so they changed their name on Medium - "not anything to be ashamed of or secret just private and personal! Felt a bit exposed". Others were concerned about negative comments and whether their blog posts would be of value to others. It is recognised that an open learning philosophy and the reflective nature of the assessment might be problematic for some learners (and educators) and such personal and emotional exposure could affect their digital wellbeing (Hibberson et al., 2020). Furthermore, Deeley and Bovill (2017) suggest that co-created assessments in the curriculum "may unearth hidden risks and anxieties for both teachers and students" and that not all staff and students view this as "an exciting new foreign landscape of assessment." These clear challenges highlight the potential risks for all involved in both open pedagogy and co-created teaching and learning. Writing on a course discussion board, one student reflected:

"I found the concept of putting my personal thoughts out there a little daunting but once I got going it was OK, in fact better than OK I had too much to say which led to the problem of cutting it down to fit the word count."

The potential for students' anxiety was considered during the design of the assessment, mostly in the context of openly publishing their personal reflections. The option of private submission or writing under a pseudonym was therefore included, in order to mitigate the risks. However, those who had expressed concern about writing on an open platform said that their feelings had 'changed' or 'somewhat changed' after the assessment, as they had received positive feedback and no negative comments, which helped alleviate their concerns. The assessment was also presented to the students as an opportunity to co-create learning materials for the benefit of their cohort and that of future students and the openness of the final assessment enabled them to use the marking framework to make a private evaluative judgement on the quality of other blog posts. Students' feedback suggested neither positive nor negative attitudes to the co-creation aspect of the assessment, although they weren't specifically asked about this through either survey. However, there is acknowledgement that providing students with opportunities to develop evaluative judgement can not only improve their understanding of assessment and feedback processes, but can also support them in becoming responsible self-regulated learners, developing skills required for within educational settings and for beyond them (Tai et al., 2018).

An assignment submitted to a closed platform and kept private would have resulted in a very different learning outcome for the students. The 'Why Public Health' publication enabled a rapid insight into the current role and life experiences of individuals on the programme. To achieve this would otherwise take weeks of 'getting to know you' discussions, even on a face-to-face course, and may never be achieved in an online course. Traditionally, these are assessments that would never have been read by anyone other than the tutor marking them. Interestingly, nine out of ten students 'agreed' or 'completely agreed' that 'reading other students' personal reflections helped them to relate to other students on the course'. Many blog posts were deeply reflective and expressive accounts of students' journeys to studying public health. One student commented:

"It's very interesting to hear what brings everyone to the course and reaffirmed we are all on different pathways and it's ok to feel a little overwhelmed!' 
Hearing reasons for why other people had chosen to study, set in the context of their work and life experiences, gave other students confidence that they had made the right choice; they consequently felt able to share their reasons and personal reflections with others. Nine out of ten students 'agreed' or 'completely agreed' that the publication 'helped them to understand the breadth of experience of all students on the course'. In her study of student and staff perceptions of partnership projects, Lubicz-Nawrocka (2018) found that "co-creation of the curriculum had a transformational effect on student participants who felt respected, valued, and more confident to contribute not only in the classroom but also in wider society." Citing Noddings (2005), she suggests that care and respect develop through safe environments where students and staff can explore new approaches to learning and teaching. Bovill (2020) adds that whole-class co-creation can help develop relationships (student-to-student and student-to-staff), offering greater opportunities for inclusive practice and potentially leading to a stronger sense of community and belonging. The publication has been shared widely across the programme and individual blog posts shared by students through social media. Some students commented that they were able to share their blog posts with friends and family and felt it helped to communicate to others what the course was all about and why they were doing it:

"I was very pleased with the positive comments about my blog - it's not normally possible to get such feedback from peers, friends and colleagues about your assignments."

Some students felt that reading other people's blog posts resonated with them, gave them insights and made them think about how they might react in similar situations. This suggests that the open, reflective nature of the assessment has created broader learning opportunities for the cohort and provided a platform for engagement with other views and experiences.

The survey responses overwhelmingly showed that the benefits of writing on an open online platform helped develop online social presence, enabling connection with others on the course and with a wider audience. This suggests the emergence of a learning community for this cohort. However, only five out of ten students 'agreed' or 'completely agreed' that 'writing about a personal experience and reading other reflections early in the course helped create a feeling of overall group cohesion'. Other elements of the course unit contributed to an overall sense of community within the cohort. This included the active engagement on the course discussion boards and the team project later in the course. Additionally, the students created their own WhatsApp groups, which provided further group support outside the formal learning environment (Greenhow and Lewin, 2016). However, the timing of the reflective assessment at the beginning of the course unit did appear to have a positive impact on the other factors that contributed to a sense of learning community, as students in the cohort were able to identify individuals on discussion boards, in webinars and in the team projects. One student said that "you felt like you had a connection with these people, even though you had never met'. Interestingly, six out of ten students 'agreed' or 'completely agreed' that they felt 'a stronger bond to students on this unit than in other units they were taking'.

\section{Conclusions}

Student feedback suggests the open, reflective nature of the assessment has improved their learning experience and contributed towards a sense of learning community through enhanced social cohesion within the group. Many students who were new to public health or who had returned to studying after a long absence were apprehensive at the start of the course unit, but felt that the blog posts had helped them find out about and empathise with each other, and explore and reflect on their personal reasons for joining the programme. This gave them confidence that they had 
made the right choice and they felt able to share their reasons and personal experiences with others. Although not the initial intention, the reflective assessment became an effective icebreaker for students on this course unit. With the current shift to online education, developing innovative pedagogies for strengthening social presence and building productive learning communities are now more important than ever.

The 'Why Public Health?' publication is an example of co-created teaching and learning both in and of the curriculum (Bovill and Woolmer, 2018; Lubicz-Nawrocka, 2019). The co-creation of the publication can benefit future student cohorts' experience. It can help to support the transition of students new to public health and postgraduate-level study. As it is an open online resource, students new to the programme can engage with these blog posts before they officially embark upon it, and the blog posts can help support online induction activities to reassure new students and help them feel part of a broader community. The publication can also help support new students in understanding assessment processes at the beginning of the course unit. For example, a formative task could require students to use the marking criteria to make evaluative judgements on previous posts and help them write their own reflections. Future cohorts of students will add to the publication each year, continuing the cycle of co-creation of teaching and learning both in and of the curriculum on this course unit. Plans are already in motion to adapt this assessment as an induction activity for a new BSc in Public Health, to start in September 2021, using the publication as a co-created element of the undergraduate curriculum. Learning activities will be designed around the publication and students will be encouraged to add their own reflections to it.

The publication has the potential to grow into a broader, open learning community of engagement with public health issues beyond the initial cohort. However, it is recognised that further work is needed to ensure that the publication is accessed beyond the assessment task, through embedding it into the broader curriculum and programme activities. For example, all staff and students on the $\mathrm{MPH}$ can contribute to the development of this resource, owing to the open nature of the assessment, to create a cross-programme collaboration. Additionally, the publication can support induction activities and marketing of the course.

This approach to assessment might well be applied to other programmes of study as an induction or icebreaker activity, where students on a course would benefit from seeing each other's contributions and where each contribution would, collectively, create a sense of cohort and community. Some academics, however, might see the open nature of the assessment as a barrier and thus not engage, especially if they were new to open assessments or not yet open practitioners. Academics who wanted to explore this method of assessment could gradually introduce more open practices into their work before exploring open approaches to assessment. The reflective element of the assessment could also be seen as problematic by some disciplines and levels of study without reflective writing in their programme or whose student body is homogeneous - by contrast with the context of this case study, where all the students had varied and unique backgrounds and motivations to study Public Health and often told fascinating personal stories about how they became interested in the topic. However, this assessment could be adapted for any type of writing (e.g., creative writing or academic writing) to suit the discipline or any situation where an open element is required. 


\section{Reference list}

Blake, J., Millson, C. and Aston, S. (2019) 'Bursting Out of the Bubble: Social Media, Openness and Higher Education.' In: Chris Rowell (ed.), Social Media in Higher Education: Case Studies, Reflections and Analysis. Cambridge, UK: Open Book Publishers. Available at: https://doi.org/10.11647/OBP.0162 (Accessed: 24 February 2021).

Bonk, C.J. (2012) 'Making a Contribution: Opener \#6: Learner Participation in Open Information Communities'. In: Bonk, C.J. (ed.), The World Is Open : How Web Technology Is Revolutionizing Education. San Francisco, CA: Jossey-Bass. ISBN: 978-1-118-01381-6

Bovill, C. and Woolmer, C. (2018) 'How conceptualisations of curriculum in higher education influence student-staff co-creation in and of the curriculum.' Higher Education, 78, 407-422. Available at: http://doi.org/10.1007/s10734-018-0349-8 (Accessed: 24 February 2021).

Bovill, C. (2020) 'Co-creation in learning and teaching: the case for a whole-class approach in higher education.' Higher Education, 79, 1023-1037. Available at: https://doi.org/10.1007/s10734018-0349-8 (Accessed: 24 February 2021).

Conole, G. (2013) Designing for Learning in an Open World. New York: Springer New York. ISBN: 978-1-4419-8517-0

Conrad, D. (2005) 'Building and maintaining community in cohort-based online learning.' International Journal of E-Learning \& Distance Education, 20(1), 1-20. Available at: http://www.ijede.ca/index.php/jde/article/view/78 (Accessed: 24 February 2021).

Deeley, S.J. and Bovill, C. (2017) 'Staff student partnership in assessment: enhancing assessment literacy through democratic practices.' Assessment \& Evaluation in Higher Education, 42(3), 463477. Available at: https://doi.org/10.1080/02602938.2015.1126551 (Accessed: 24 February 2021).

Greenhow, C. and Lewin, C. (2016) 'Social media and education: Reconceptualizing the boundaries of formal and informal learning.' Learning, Media and Technology, 41, 6-30. Available at: http://doi.org/10.1080/17439884.2015.1064954 (Accessed: 24 February 2021).

Healey, M., Flint, A., and Harrington, K. (2014) ‘Engagement through partnership: Students as partners in learning and teaching in higher education.' Available at: https://www.advancehe.ac.uk/knowledge-hub/engagement-through-partnership-students-partners-learning-and-teachinghigher (Accessed: 24 February 2021).

Hibberson, S., Barrett, E. and Kelly, J. (2020) 'Digital Wellbeing of Learners.' Available at: https://www.jisc.ac.uk/guides/digital-wellbeing-of-learners (Accessed: 24 February 2021).

Johnston, S. and Stewart, B. (2020) 'The Open Page: A Case Study of Partnership as Open Pedagogy.' International Journal for Students As Partners, 4(2), 81-89. Available at: https://doi.org/10.15173/ijsap.v4i2.4182 (Accessed: 24 February 2021).

Kear, K. (2010) 'Feeling Connected.' In: Kear, K. (ed.), Online and Social Networking Communities: A Best Practice Guide for Educators. London: Taylor \& Francis Group. ISBN: 9780415872478 
Kear, K., Chetwynd, F. and Jefferis, H. (2014) 'Social presence in online learning communities: the role of personal profiles.' Research in Learning Technology, 22. Available at:

https://doi.org/10.3402/rlt.v22.19710 (Accessed: 24 February 2021).

Lubicz-Nawrocka, T.M. (2018) 'Students as partners in learning and teaching: The benefits of cocreation of the curriculum.' International Journal for Students as Partners, 2(1), 47-63. Available at: https://doi.org/10.15173/ijsap.v2i1.3207 (Accessed: 24 February 2021).

Lubicz-Nawrocka, T.M. (2019) 'More than just a student: How co-creation of the curriculum fosters third spaces in ways of working, identity, and impact.' International Journal for Students as Partners, 3(1), 34-49. Available at: https://doi.org/10.15173/ijsap.v3i1.3727 (Accessed: 24 February 2021).

Noddings, N. (2005) The challenge to care in schools: An Alternative Approach to Education (2nd edition). New York: Teachers College Press. ISBN: 0-8077-4609-6.

Tai, J., Ajjawi, R., Boud, D., Dawson, P. and Panadero, E. (2018) 'Developing evaluative judgement: enabling students to make decisions about the quality of work.' Higher Education, 76, 467-481. Available at: https://link.springer.com/article/10.1007/s10734-017-0220-3 (Accessed: 24 February 2021). 


\section{Appendix 1 - Online survey}

1. Did you add your reflective post to the 'Why Public Health?' publication?
a. Yes
b. No

2. What was the reason you didn't add your reflective post to the publication?

3. How did you feel writing about a personal experience on an open online platform? (Select the one that mostly summarises your experience)

a. I didn't feel any apprehensiveness writing about a personal experience on an open online platform

b. I had no problems writing about a personal experience however I was concerned about posting it on an open online platform

c. I was mostly worried writing about a personal experience rather than posting on an open online platform

d. I felt very apprehensive writing about a personal experience on an open online platform

4. Please elaborate on any concerns you had about either writing about a personal experience and/or posting on an open online platform.

5. Did your feelings change after completing the reflective blog post?
a. Yes
b. No

6. Please elaborate on what changed after completing the reflective post.

7. What do you think were the benefits of writing on an open online platform?

8. Were there any negatives to writing on an open online platform?

9. To what extent do you agree with the following statements...

a. Reading other students' personal reflections helped me to relate to other students on the course

b. The 'Why Public Health' publication helped me to understand the breadth of experience of all students on the course

c. Writing about a personal experience and reading other reflections early in the course helped create a feeling of overall group cohesion

d. Writing about a personal experience early in the course has encouraged me to engage more with the discussion tasks in the course

e. Reading other students' personal reflections has helped me bond with teammates in the group project assessment

f. I feel a stronger bond to students on this unit than in other units I am taking (if applicable)

10. In your opinion, did anything else impact on the cohesion of the group? 